\title{
Procalcitonin and lung ultrasonography point-of-care testing to decide on antibiotic prescription in patients with lower respiratory tract infection in primary care: protocol of a pragmatic cluster randomized trial
}

Loïc Lhopitallier ${ }^{1 *}$ (D) Andreas Kronenberg ${ }^{2}$, Jean-Yves Meuwly ${ }^{3}$, Isabella Locatelli ${ }^{4}$, Julie Dubois ${ }^{5}$, Joachim Marti ${ }^{5}$, Yolanda Mueller ${ }^{6}$, Nicolas Senn ${ }^{6}$, Valérie D'Acremont ${ }^{4,7}$ and Noémie Boillat-Blanco ${ }^{1}$

\begin{abstract}
Background: A minority of patients presenting with lower respiratory tract infection (LRTI) to their general practitioner (GP) have community-acquired pneumonia (CAP) and require antibiotic therapy. Identifying them is challenging, because of overlapping symptomatology and low diagnostic performance of chest X-ray. Procalcitonin (PCT) can be safely used to decide on antibiotic prescription in patients with LRTI. Lung ultrasound (LUS) is effective in detecting lung consolidation in pneumonia and might compensate for the lack of specificity of PCT.

We hypothesize that combining PCT and LUS, available as point-of care tests (POCT), might reduce antibiotic prescription in LRTIs without impacting patient safety in the primary care setting.

Methods: This is a three-arm pragmatic cluster randomized controlled clinical trial. GPs are randomized either to PCT and LUS-guided antibiotic therapy or to PCT only-guided therapy or to usual care. Consecutive adult patients with an acute cough due to a respiratory infection will be screened and included if they present a clinical pneumonia as defined by European guidelines. Exclusion criteria are previous antibiotics for the current episode, working diagnosis of sinusitis, severe underlying lung disease, severe immunosuppression, hospital admission, pregnancy, inability to provide informed consent and unavailability of the GP. Patients will fill in a 28 day-symptom diary and will be contacted by phone on days 7 and 28. The primary outcome is the proportion of patients prescribed any antibiotic up to day 28. Secondary outcomes include clinical failure by day 7 (death, admission to hospital, absence of amelioration or worsening of relevant symptoms) and by day 28 , duration of restricted daily activities, episode duration as defined by symptom score, number of medical visits, number of days with side effects due to antibiotics and a composite outcome combining death, admission to hospital and complications due to LRTI by day 28. An evaluation of the cost-effectiveness and of processes in the clinic using a mixed qualitative and quantitative approach will also be conducted.

(Continued on next page)
\end{abstract}

\footnotetext{
* Correspondence: loic.lhopitallier@chuv.ch

${ }^{1}$ Infectious Diseases Service, University Hospital of Lausanne, Lausanne,

Switzerland

Full list of author information is available at the end of the article
}

C The Author(s). 2019 Open Access This article is distributed under the terms of the Creative Commons Attribution 4.0 International License (http://creativecommons.org/licenses/by/4.0/), which permits unrestricted use, distribution, and reproduction in any medium, provided you give appropriate credit to the original author(s) and the source, provide a link to the Creative Commons license, and indicate if changes were made. The Creative Commons Public Domain Dedication waiver (http://creativecommons.org/publicdomain/zero/1.0/) applies to the data made available in this article, unless otherwise stated. 
(Continued from previous page)

Discussion: Our intervention targets only patients with clinically suspected CAP who have a higher pretest probability of definite pneumonia. The intervention will not substitute clinical assessment but completes it by introducing new easy-to-perform tests.

Trial registration: The study was registered on the 19th of June 2017 on the clinicaltrials.gov registry using reference number; NCT03191071.

Keywords: Lower respiratory tract infections, Procalcitonin, Lung ultrasound, Antibiotic prescription, General practice, Point of care testing,

\section{Background}

As a clear association exists between antibiotic use and resistance rates at community and patient levels, reducing inappropriate use is essential $[1,2]$. The highest volume of prescriptions occurs in outpatients presenting with acute respiratory infections (ARIs) [3, 4]. Amongst these, lower respiratory tract infections (LRTIs) are the commonest acute reasons for consulting general practitioners (GPs) [5] LRTIs include acute bronchitis, exacerbation of chronic obstructive pulmonary disease (COPD) and community-acquired pneumonia (CAP). CAP in adults is associated with high morbidity and mortality rates and requires antibiotic treatment [6]. Whereas only 5 to $12 \%$ of patients presenting to their GP with LRTIs have CAP, $60 \%$ receive an antibiotic prescription $[7,8]$.

The absence of specific signs and symptoms for CAP makes identifying these patients a challenge [9]; in this context, the presence of a new infiltrate on chest X-ray supports the definite diagnosis [10]. However, chest X-ray has several limitations: it is not always readily available at the GP level $[11,12]$, exposes patients to radiation and has a limited diagnostic accuracy for CAP (54\% sensitivity and $57 \%$ specificity, using chest-CT as a gold standard) [13].

Several studies have evaluated the use of host biomarkers to help physicians identify patients with respiratory tract infections potentially requiring antibiotics. Procalcitonin (PCT) and $\mathrm{C}$ reactive protein (CRP) are the most extensively studied. PCT is more sensitive than CRP in differentiating bacterial from viral infections in ARIs (respectively 92 and $86 \%$ ), for a similar specificity (respectively 73 and 70\%) [14]. In various settings, including primary care, the use of PCT to guide antibiotic prescription in ARIs reduces antibiotic treatment without affecting outcome [11, 15-17]. Its low specificity in differentiating bacterial from viral aetiologies limits its use in settings with high rates of viral infections, such as primary care. More data are required to confirm the impact of PCT-guided therapy on antibiotic prescription rates among patients with LRTIs in primary care.

Lung ultrasound is effective in detecting lung consolidation and has a 92\% sensitivity and 93\% specificity using chest-CT as a gold standard for the diagnosis of CAP $[13,18,19]$. GPs or non-physician respiratory therapists can perform and interpret lung ultrasound after a short training course $[20,21]$. The maximum exam duration is of $13 \mathrm{~min}$ per patient [22]. The high specificity of lung ultrasound to detect lung consolidation could potentially compensate the low specificity of PCT.

We hypothesize that the combination of lung ultrasound and PCT should improve the accuracy of the diagnosis of CAP in primary care. To reduce unnecessary antibiotic prescription without affecting patient safety, we plan to test a novel simple clinical management algorithm (UltraPro) integrating the results of procalcitonin and lung ultrasound used as point-of-care tests (POCT).

\section{Methods/design Study objectives}

The main objective of the study is to evaluate if the UltraPro algorithm based on procalcitonin and lung ultrasound decreases antibiotic prescription amongst adult patients with LRTIs managed at primary care level in Switzerland compared to the use of PCT only and usual care.

The secondary objectives are to compare the clinical outcome of patients in each arm, to evaluate the acceptability and feasibility of the intervention and to calculate and compare cost-effectiveness between arms.

\section{Study design and setting}

This is a three-arm pragmatic cluster randomized controlled superiority trial conducted in GPs practices.

GPs in 6 cantons in Western and central-Western Switzerland (Bern, Vaud, Neuchâtel, Fribourg, Valais and Jura) were recruited to ensure representation of different cultural areas and antibiotic prescription rates [23]. We chose these regions due to good collaboration between the investigators and a strong network of GPs during previous studies, which is essential for the feasibility of the study [24, 25]. The list of the participating GPs can be found in Additional file 1: Table S1. 


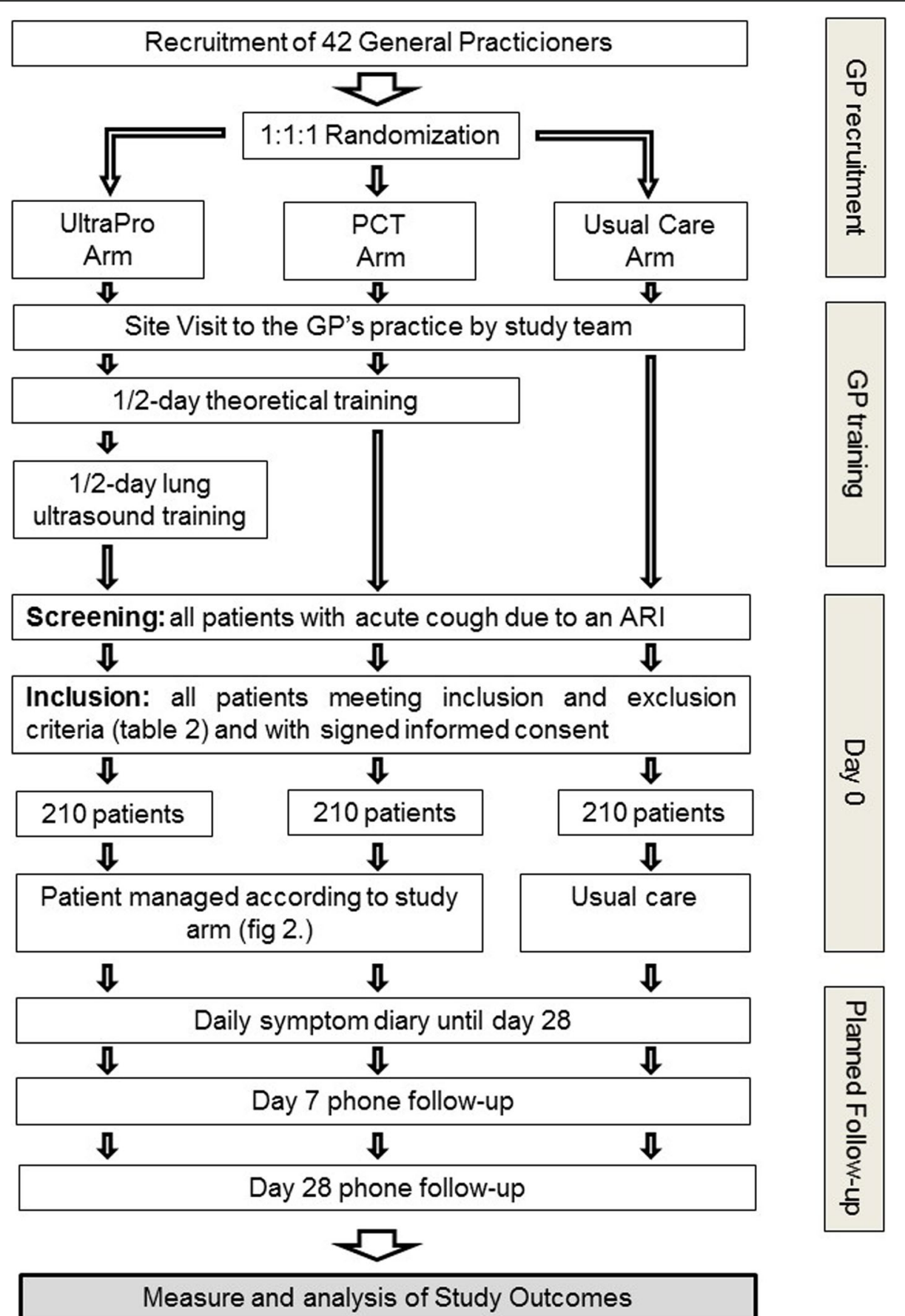

Fig. 1 Study design of the randomized intervention study. Abbreviations: PCT: procalcitonin, GPs: general practitioners, ARI: acute respiratory infection, LRTI: lower respiratory tract infection

\section{Randomization}

We will randomly allocate GPs to one of the three arms of the study: UltraPro, PCT only or usual care. Randomization with a $1: 1: 1$ ratio between arms using a computer generated list with variable block sizes will be performed using the REDCap $\odot$ electronic data capture and management tool [26]. Due to the nature of the intervention, GPs are not blinded.

\section{Outcomes and hypotheses}

The primary outcome is the difference in the proportion of patients prescribed an antibiotic in each arm by day 28. Table 1 summarizes the secondary outcomes. 
Table 1 Study outcomes

Primary outcome measure

Proportion of patients prescribed an antibiotic in each arm by day 28

Secondary outcome measures

Clinical outcomes

By day 7

Proportion of patients with clinical failure, defined as:

- admission to hospital OR

- death OR

- absence of amelioration or worsening of relevant symptoms (fever and/or dyspnoea)

$$
\text { By day } 28
$$

Proportion of patients with an adverse outcome, defined as:

- admission to hospital OR

- death OR

complications due to LRTI (persistence of pneumonia, lung abscess,

lung effusion, empyema or sepsis)

Duration of restricted daily activities due to a respiratory tract infection

Duration of the episode (defined by the total daily symptom score)

Number of medical visits for the episode of LRTI

Number of days with side effects related to antibiotics

Consultation process outcomes

- Time spent by the patient in the practice, time required for the whole consultation

- Patient satisfaction with diagnostic process and consultation outcome

- Quality of the ultrasound images and of their interpretation

- Provider adhesion, level of trust and perceived usefulness of the algorithm recommendation

- Identification of barriers and facilitators to the implementation of

UltraPro algorithm in primary care

Economic outcomes

Cost / effectiveness ratio

\section{Participants}

\section{General practitioners}

GPs known to the investigators for their interest in participating in research and members of local medical societies will be contacted. Study advertising will also be published in GP's medical journals.

GPs are eligible if they do not use any of the UltraPro POCT tests for the management of their patients with a LRTI in their routine practice. To avoid contamination between arms, only one GP per practice will be included. Each participating GP will be responsible for enrolling 15 patients for a maximum period of 15 months.

\section{Patients}

GPs will screen for inclusion all adult patients (aged 18 years or older) presenting with a cough due to upper and/or lower respiratory tract infections. They will include every consecutive patient meeting the inclusion and exclusion criteria (Table 2) and providing informed consent (Fig. 1). GPs are responsible for obtaining informed consent.

\section{Study intervention}

\section{Arm 1: UltraPro}

The UltraPro algorithm (Fig. 2) combines the results of a PCT point-of-care test with a lung ultrasound to decide on antibiotic prescription. The medical assistant will measure PCT using the portable Thermo-Fisher $\odot$ PCT Direct rapid point-of-care test. This immunoassay provides a quantitative PCT result in 20 min using $20 \mu \mathrm{L}$ of whole blood. This new device has been validated by comparison with the reference method (Elecsys ${ }^{\odot}$ and Kryptor $^{\oplus}$ B.R.A.H.M.S. PCT assays) with an excellent correlation index (Pearson's log-r, $r^{2}=0.95$ ) [27]. The measuring range for whole blood samples is 0.22 to $10 \mu \mathrm{g} / \mathrm{l}$. According to previous studies, a $0.25 \mu \mathrm{g} / \mathrm{L}$ threshold is safe to decide on antibiotic prescription for ARIs at primary care level $[11,17,28]$.

Table 2 Inclusion and exclusion criteria

\begin{tabular}{ll}
\hline Inclusion criteria [10] & Exclusion criteria \\
\hline $\begin{array}{l}\text { acute cough }(<21 \text { days) and at least one of the } \\
\text { following sign/symptom: }\end{array}$ & previous prescription of antibiotics for the current episode \\
$\begin{array}{l}\text { • history of fever for more than } 4 \text { days } \\
\text { - dyspnoea }\end{array}$ & working diagnosis of acute sinusitis or of a non-infective disorder \\
- tachypnoea ( $>22$ cycles per minute) & previous episode of chronic obstructive pulmonary disease exacerbation treated with antibiotics \\
- abnormal focal finding upon lung & during the last 6 months \\
auscultation & known pregnancy \\
& severe immunodeficiency (untreated HIV infection with CD4 count $<200$ cells $/ \mathrm{mm}^{3}$, solid organ \\
& transplant receiver, neutropenia $(<1000$ cells $/ \mu l)$, treatment with corticosteroids (dose equivalent \\
& to 20 mg prednisone/day for $>28$ days) \\
& decision by the GP to admit the patient \\
& GP not available for performing study \\
& patient unable to provide informed consent \\
\hline
\end{tabular}




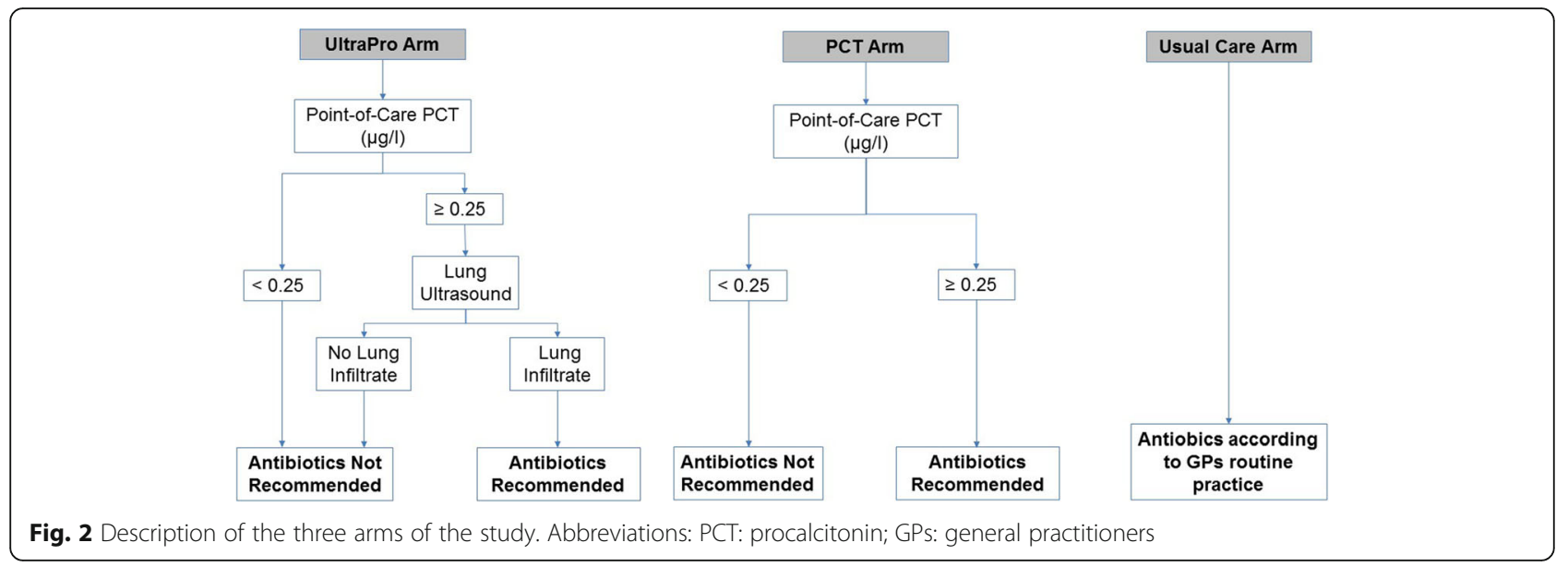

In case of an elevated PCT $(\geq 0.25 \mu \mathrm{g} / \mathrm{L})$, the GP will perform a lung ultrasound with a portable L12-4 convex transducer (Philips $\odot$ Lumify) to look for the presence of a lung consolidation (Fig. 2). Lung consolidations are defined as sub-pleural echo-poor regions, sub-pleural regions with a tissue-like echotexture or a focal increase of $B$ lines [29]. The procedure will follow international evidence-based recommendations for point-of-care lung ultrasound [29, 30]. We expect the examination to last 10 to $15 \mathrm{~min}$.

Only in the presence of an elevated PCT and a lung consolidation will the algorithm recommend antibiotics (Fig. 2). The GP will choose the antibiotic, its dosage and the duration of treatment. The GP is free to order additional diagnostic tests.

\section{Arm 2: PCT only}

The medical assistant will measure PCT using the POCT assay described above. Only in the presence of an elevated PCT will the algorithm recommend antibiotics (Fig. 2). The GP will choose the type of antibiotic, its dosage and the duration of treatment. The GP is free to order additional diagnostic tests.

\section{Arm 3: usual care}

GPs will manage and treat these patients as they usually do (Fig. 2).

\section{Physician's training}

GPs in the UltraPro and PCT arms will participate in a half-day training session. Topics will include antibiotic resistance, epidemiology of pneumonia in Switzerland, management of CAP in primary care, the use of PCT and lung ultrasound to guide antibiotic prescription, the rationale for the UltraPro algorithm and the procedures of the study.

GPs in the UltraPro arm will participate in an additional lung ultrasonography half-day training session to achieve independent practice and appropriately identify lung consolidation. Topics include ultrasound physics, ultrasound equipment, probe positioning, image recording and interpretation using a phantom simulator (CAE Healthcare $\odot$ ). GPs will take a practical exam at the end of the training session. Three to five months following the start of the study, we will conduct an extra $2 \mathrm{~h}$ lung ultrasonography training session with inpatients admitted with CAP.

Before patient recruitment, the study team will visit all GPs at their own practices and train medical assistants in POCT PCT measurement.

GPs in the usual care arm will receive a two-hour training on the rationale and the technical procedures of the study. There will be no training on epidemiology and management of pneumonia.

\section{Data collection and monitoring}

Data collected at inclusion by the GPs include demographics, co-morbidities, symptoms and their respective duration, vital and other clinical signs, laboratory tests and radiologic exams ordered outside the scope the algorithm, PCT result, lung ultrasound interpretation and antibiotic prescription. GPs will record all ultrasound images along with relevant metadata.

Study data will be collected via an electronic case report form (eCRF) and managed using REDCap ${ }^{\text {TM }}$ electronic data capture tools hosted at Lausanne University Hospital [26]. In the UltraPro and PCT arms, medical assistants will draw venous blood to perform the PCT POCT measurement.

The study coordinator will perform data monitoring under guidance from the steering committee that is composed of the principal investigator, the co-principal investigators and the co-investigators. The Clinical Trial Unit of the University Hospital of Lausanne will perform the external monitoring of the study. 


\section{Follow-up}

A member of the study team (blinded to study arm) will conduct a standardized phone interview of all participants on day 7 and day 28 . He will record clinical outcomes (presence or recurrence of LRTIs symptoms), additional medical visits, additional antibiotic prescription, number of days during which activities (work or recreation) were restricted, antibiotic side effects, secondary hospital admission and patient satisfaction.

All participants will fill a validated daily diary until symptom resolution, up to a maximum of 28 days [31]. They will report each day on six items (cough, phlegm, shortness of breath, sleep disturbance, impairment of normal daily activities and feeling unwell) on a Likert scale (1-6). By summing the values, we will obtain a daily composite symptom score for each patient.

In case of follow-up visits, GP's will manage their patients according to their habitual practice.

\section{Analyses \\ Sample size calculation}

We calculated the sample size to assess an absolute difference in antibiotic prescription of at least 15\% between each study arm. The steering committee considered this difference as sufficient to warrant implementation of the intervention at a larger scale. Sixty \% of patients are estimated to receive antibiotics with usual care [8]; we expect it to be $45 \%$ using PCT only and $30 \%$ combining PCT and lung ultrasound.

A study sample of 14 GPs and 15 patients per GP in each arm (210 patients per arm for a total of 630 patients) gives a power of $80 \%$ to detect the expected difference in antibiotic prescription with $5 \%$ level of significance, when adjusting for clustering at practice level (intracluster coefficient 0.06) [8]. This sample size also guarantees a power of $80 \%$ to prove non-inferiority in terms of duration of activities restriction (non-inferiority margin of 1 day, standard deviation of 4 days) as well as in terms of the proportion of patients with adverse outcome by day 28 with an estimated probability in the "usual care" arm of 0.05 (non-inferiority margin of 0.02 ).

\section{Statistical analyses}

The population of all patients included, irrespective of follow-up will be used for primary data analysis. For patients who are lost to follow-up we will consider them as having had clinical failure, an adverse outcome and a duration of disease to be equal to the maximum measured in other patients. We will exclude patients whose GPs did not follow the algorithm recommendation and/ or who did not complete telephone follow-up of the perprotocol analysis.

The primary analysis will be a logistic regression corrected for variation at GP level (generalized linear mixed effect) to calculate the difference in the proportion of patients prescribed an antibiotic by day 28 as well as the odds ratio of antibiotic prescription between 2 groups.

We will compare secondary outcomes (clinical, consultation process and economic outcomes) between groups using linear mixed effect regression. We will compare episode duration between groups using survival analysis methods.

\section{Qualitative evaluation}

Qualitative assessment will be done by face to face semistructured interviews with a subset of the participating GPs. Focus groups will also be conducted with a subset of the medical assistants. Interview guides for both the interviews and focus group will focus on needs for training, feasibility of patient's recruitment and comfort with intervention procedures. We will record all semi-structured interviews and focus groups using standard equipment, transcribed, and coded. Using a content analysis approach, the transcriptions will be firstly analysed to identify key themes and to develop a coding frame. Two different investigators will code independently each transcript and compare them for agreement. The coded data will be analysed and interpreted with regard to the identification of barriers and facilitators to the implementation of interventions processes.

\section{Economic evaluation}

We will conduct a within-trial economic evaluation and complement it with a longer-term economic model. All resources involved in the use of the UltraPro algorithm will be included as intervention costs. These include: 1) human resources: time spent training GPs and medical assistants, additional consultation time and additional time spent by the medical assistant measuring point-of-care procalcitonin, 2) use of health services: referrals to hospital, hospitalizations, unplanned or planned GP consultations, 3) medical supplies and equipment: consumables, investment in devices (PCT reader, ultrasonography transducer).

The eCRF will provide most of the data to measure resource use. Resource use will be valued using the appropriate unit cost, in Swiss Francs (CHF) (e.g. wage rate, tariff, etc.). The within-trial analysis will involve calculating the incremental cost-effectiveness ratio, expressed in CHF per percentage point reduction in antibiotic prescription using the UltraPro algorithm as opposed to PCT only and usual care. In order to incorporate the potential wider benefits of a reduction in antibiotics prescriptions, such as reduced anti-microbial resistance, we will design a long-term economic model.

\section{Time plan for the study}

Patient recruitment will begin in September 2018 and last until February 2020. 


\section{Discussion}

This will be the first trial to assess POCT PCT measurement as well as its combination with lung ultrasonography for deciding on antibiotic prescription in patients with LRTIs presenting to primary care. Frequent inappropriate antibiotic prescription in this population is in part due to diagnostic uncertainties surrounding the non-specific clinical presentation of patients with LRTIs and to the low performance of available radiological tools. Physicians need better and easily implemented POCT tools to help them decide on antibiotic prescription in this population.

Our study has several strengths. The chosen randomization level, i.e. GP level, will minimize bias by avoiding contamination between arms that could occur if we randomized at patient level. The screening strategy targeting all patients with cough due to an ARI will allow us to evaluate the proportion of patients with ARIs in whom CAP is suspected and the overall proportion of ARI patients receiving antibiotics. These data will allow us to grasp the potential impact of our intervention from a public health perspective. The inclusion criteria are the clinical criteria for suspecting CAP according to European guidelines. These stringent inclusion criteria guarantee the reproducibility of the study in various settings and avoid using the GP's subjective clinical diagnosis, as done in previous studies [17]. They also help clinicians in real-life practice target patients who will benefit from additional diagnostic tests after their clinical evaluation. As reflected by our inclusion criteria, our intervention targets only patients with a higher pre-test probability of definite pneumonia. We chose not to include patients with acute bronchitis or asthma exacerbation as it is established that such patients do not require antibiotics [32].

The intervention should not substitute clinical assessment; it completes it by introducing new easy-to-perform tests in case of suspected CAP. Recent studies that did not show an impact of PCT-guided antibiotic prescription in LRTIs included patients, for whom antibiotics were not indicated on clinical grounds alone, thus diluting any potential effect of the intervention [33].

There will be exclusion of a well-defined subset of comorbid patients (severe underlying lung disease, severe immunosuppression, etc. ...) who when presenting with an LRTI should receive antibiotics regardless of clinical presentation, as there are no data showing that they can be safely managed using procalcitonin and lung ultrasound.

As this is a pragmatic trial, the chosen comparator group is "usual care" rather than standard of care. We intend to compare our intervention to real life clinical practice and clinicians in the usual care arm will manage their patient as per their habitual practice. We consider that GPs are aware of existing clinical guidelines and that there are practical and/or pragmatic reasons for not following recommendations.

There will be a PCT only arm. Although PCT is a sensitive test that can safely reduce antibiotic consumption among patients with ARIs $[11,16]$, more data are needed to confirm its impact on antibiotic prescription rates when compared to routine setting amongst primary care patients with LRTIs. We did not include an additional arm testing lung ultrasound alone as the PCT pre-screening to decide on lung ultrasonography will help save time whilst managing the patients and will be easier to implement at a larger scale in GP practices.

Our study has some limitations. Although we are conducting a pragmatic trial, GP participation is on a voluntary basis, leading to inclusion bias. We expect these motivated and informed GP to have low rates of inadequate antibiotic prescription. This could reduce the effect of our intervention. A reduction in antibiotic prescription rates in this group could then suggest that the real world effect would be greater. We also expect that there will be some deviations from the recommendations of the PCT only and UltraPro algorithms. To reduce the risk of overruling, we present the rationale behind the algorithms to the GPs during the half-day training session and exclude patients who should anyway receive antibiotics when presenting with an LRTI. The possibility of overruling increases the applicability of the findings.

PCT and lung ultrasound are now readily available as POCT making them easy to implement in primary care practices. Use rates of point-of-care ultrasonography in GP practices is variable in Europe [34], it is estimated that around $30 \%$ of Swiss GPs have an ultrasound machine at their practice (https://www.doctorfmh.ch/) [35] . This proportion will probably further increase in a near future due to the development of portable and affordable machines together with available short training courses. A pilot study done between December 2017 and April 2018 showed that the implementation of both tools in the proposed setting was feasible. Decentralization of the laboratory analyses and radiologic examination to the GPs practices allows fast and efficient management of patients with LRTIs, and we believe that both PCT and lung ultrasound have a role to play in helping physicians prescribe antibiotics adequately in LRTIs.

\section{Additional file}

Additional file 1: Table S1. List of participating GPs. (DOCX 13 kb)

Abbreviations

ARI: Acute respiratory infection; CAP: Community acquired pneumonia; CHF: Swiss francs; COPD: Chronic obstructive pulmonary disease; CRP: C reactive protein; eCRF: Electronic case report form; GP: General practitioner; 
LRTI: Lower respiratory tract infection; PCT: Procalcitonin; POCT: Point of care testing

\section{Acknowledgements}

We thank all the GPs, medical assistants and patients who took part in the pilot study.

\section{Authors' contributions}

NBB and LL conceived the study. NBB, LL, VDA, NS and AK participated in study design. $L L$ wrote the manuscript. JYM, JD, IL JM and YM did a critical review of the study protocol and of the manuscript. All authors have read and approved the manuscript.

\section{Funding}

The study is entirely financed by grant 407240_167133/1 of the Swiss National Science Foundation's National Research Program 72 on antimicrobial resistance. The Swiss National Science Foundation played no role in study design, development, data collection or in the generation of this manuscript. There is no other financial support.

\section{Availability of data and materials}

Not applicable.

\section{Ethics approval and consent to participate}

The ethics committee of cantons Vaud and Bern in Switzerland approved the protocol under the number 2017-01246. This approval covers all study sites. Written consent will be obtained from all study participants.

\section{Consent for publication}

Not applicable.

\section{Competing interests}

The authors declare that they have no competing interests.

\section{Author details}

${ }^{1}$ Infectious Diseases Service, University Hospital of Lausanne, Lausanne, Switzerland. ${ }^{2}$ Institute for Infectious Diseases, University of Bern, Bern, Switzerland. ${ }^{3}$ Department of Radiology, University Hospital of Lausanne, Lausanne, Switzerland. ${ }^{4}$ Department of Outpatient Care and Community Medicine, Unisanté, University of Lausanne, Lausanne, Switzerland. ${ }^{5}$ Institute of Social and Preventive Medicine, Unisanté, University of Lausanne, Lausanne, Switzerland. ${ }^{6}$ Institute of Family Medicine, Department of Outpatient Care and Community Medicine, Unisanté, University of Lausanne, Lausanne, Switzerland. ${ }^{7}$ Swiss Tropical and Public Health Institute, University of Basel, Basel, Switzerland.

\section{Received: 20 February 2019 Accepted: 15 July 2019}

Published online: 06 August 2019

\section{References}

1. Costelloe C, Metcalfe C, Lovering A, Mant D, Hay AD. Effect of antibiotic prescribing in primary care on antimicrobial resistance in individual patients: systematic review and meta-analysis. BMJ. 2010;340:c2096.

2. Goossens H, Ferech M, Vander Stichele R, Elseviers M. Outpatient antibiotic use in Europe and association with resistance: a cross-national database study. Lancet. 2005;365(9459):579-87.

3. Hurlimann D, Limacher A, Schabel M, Zanetti G, Berger C, Muhlemann K Kronenberg A, Swiss Sentinel Working G. Improvement of antibiotic prescription in outpatient care: a cluster-randomized intervention study using a sentinel surveillance network of physicians. J Antimicrob Chemother. 2015;70(2):602-8.

4. Health FOoP: Joint report 2016. Usage of antibiotics and occurrence of antibiotic resistance in Bacteria from humans and animals in Switzerland. 2016.

5. Schappert SM, Burt CW. Ambulatory care visits to physician offices, hospital outpatient departments, and emergency departments: United States, 200102. Vital Health Stat. 2006;13(159):1-66.

6. Prina E, Ranzani OT, Torres A. Community-acquired pneumonia. Lancet. 2015;386(9998):1097-108.
7. Huchon GJ, Gialdroni-Grassi G, Leophonte P, Manresa F, Schaberg T, Woodhead M. Initial antibiotic therapy for lower respiratory tract infection in the community: a European survey. Eur Respir J. 1996;9(8):1590-5.

8. Cals JW, Butler CC, Hopstaken RM, Hood K, Dinant GJ. Effect of point of care testing for $C$ reactive protein and training in communication skills on antibiotic use in lower respiratory tract infections: cluster randomised trial. BMJ. 2009;338:b1374.

9. Metlay JP, Kapoor WN, Fine MJ. Does this patient have community-acquired pneumonia? Diagnosing pneumonia by history and physical examination. JAMA. 1997:278(17):1440-5.

10. Woodhead M, Blasi F, Ewig S, Garau J, Huchon G, leven M, Ortqvist A, Schaberg T, Torres A, van der Heijden G, et al. Guidelines for the management of adult lower respiratory tract infections--full version. Clin Microbiol Infect. 2011;17(Suppl 6):E1-59.

11. Burkhardt O, Ewig S, Haagen U, Giersdorf S, Hartmann O, Wegscheider K, Hummers-Pradier E, Welte T. Procalcitonin guidance and reduction of antibiotic use in acute respiratory tract infection. Eur Respir J. 2010; 36(3):601-7

12. Briel M, Young J, Tschudi $P$, Hersberger KE, Hugenschmidt C, Langewitz W, Bucher HC. Prevalence and influence of diagnostic tests for acute respiratory tract infections in primary care. Swiss Med Wkly. 2006;136(15-16): 248-53.

13. Ye $X$, Xiao H, Chen B, Zhang S. Accuracy of lung ultrasonography versus chest radiography for the diagnosis of adult community-acquired pneumonia: review of the literature and meta-analysis. PLoS One. 2015; 10(6):e0130066.

14. Simon L, Gauvin F, Amre DK, Saint-Louis P, Lacroix J. Serum procalcitonin and C-reactive protein levels as markers of bacterial infection: a systematic review and meta-analysis. Clin Infect Dis. 2004;39(2):206-17.

15. Schuetz P, Muller B, Christ-Crain M, Stolz D, Tamm M, Bouadma L, Luyt CE, Wolff $M$, Chastre J, Tubach F, et al. Procalcitonin to initiate or discontinue antibiotics in acute respiratory tract infections. Cochrane Database Syst Rev. 2012;9:CD007498.

16. Schuetz P, Wirz Y, Sager R, Christ-Crain M, Stolz D, Tamm M, Bouadma L, Luyt CE, Wolff M, Chastre J, et al. Effect of procalcitonin-guided antibiotic treatment on mortality in acute respiratory infections: a patient level metaanalysis. Lancet Infect Dis. 2018;18(1):95-107.

17. Briel M, Schuetz P, Mueller B, Young J, Schild U, Nusbaumer C, Periat P, Bucher HC, Christ-Crain M. Procalcitonin-guided antibiotic use vs a standard approach for acute respiratory tract infections in primary care. Arch Intern Med. 2008;168(18):2000-7 discussion 2007-2008.

18. Cortellaro F, Colombo S, Coen D, Duca PG. Lung ultrasound is an accurate diagnostic tool for the diagnosis of pneumonia in the emergency department. Emerg Med J. 2012;29(1):19-23.

19. Lichtenstein DA, Meziere GA. Relevance of lung ultrasound in the diagnosis of acute respiratory failure: the BLUE protocol. Chest. 2008;134(1):117-25.

20. Bornemann P, Jayasekera N, Bergman K, Ramos M, Gerhart J. Point-of-care ultrasound: coming soon to primary care? J Fam Pract. 2018;67(2):70-80.

21. See KC, Ong V, Wong SH, Leanda R, Santos J, Taculod J, Phua J, Teoh CM. Lung ultrasound training: curriculum implementation and learning trajectory among respiratory therapists. Intensive Care Med. 2016;42(1):63-71.

22. Chavez MA, Shams N, Ellington LE, Naithani N, Gilman RH, Steinhoff MC, Santosham M, Black RE, Price C, Gross M, et al. Lung ultrasound for the diagnosis of pneumonia in adults: a systematic review and meta-analysis. Respir Res. 2014;15:50.

23. Achermann R, Suter K, Kronenberg A, Gyger P, Muhlemann K, Zimmerli W, Bucher HC. Antibiotic use in adult outpatients in Switzerland in relation to regions, seasonality and point of care tests. Clin Microbiol Infect. 2011;17(6):855-61.

24. Senn N, Monod S. Development of a comprehensive approach for the early diagnosis of geriatric syndromes in general practice. Front Med. 2015;2:78.

25. Kronenberg A, Blüttikofer L, Battaglia M, Frey $P$, Täuber $M$, Jüni $P$. Symptomatic therapy of uncomplicated lower urinary tract infections in the ambulatory setting. A randomized, double blind trial (NCT01039545). Copenhagen: Oral presentation, ESCMID; 2015. p. 25.

26. Harris PA, Taylor R, Thielke R, Payne J, Gonzalez N, Conde JG. Research electronic data capture (REDCap)--a metadata-driven methodology and workflow process for providing translational research informatics support. J Biomed Inform. 2009:42(2):377-81.

27. ThermoScientific: B.R.A.H.M.S PCT DIRECT Instruction for Use (Version R04en). 2018.

28. Schuetz P, Christ-Crain M, Thomann R, Falconnier C, Wolbers M, Widmer I, Neidert S, Fricker T, Blum C, Schild U, et al. Effect of procalcitonin-based 
guidelines vs standard guidelines on antibiotic use in lower respiratory tract infections: the ProHOSP randomized controlled trial. JAMA. 2009;302(10): 1059-66.

29. Volpicelli G, Elbarbary M, Blaivas M, Lichtenstein DA, Mathis G, Kirkpatrick AW, Melniker L, Gargani L, Noble VE, Via G, et al. International evidencebased recommendations for point-of-care lung ultrasound. Intensive Care Med. 2012;38(4):577-91.

30. Rambhia SH, D'Agostino CA, Noor A, Villani R, Naidich JJ, Pellerito JS. Thoracic ultrasound: technique, applications, and interpretation. Curr Probl Diagn Radiol. 2017;46(4):305-16.

31. Watson L, Little P, Moore M, Warner G, Williamson I. Validation study of a diary for use in acute lower respiratory tract infection. Fam Pract. 2001;18(5): 553-4.

32. Little P, Stuart B, Moore M, Coenen S, Butler CC, Godycki-Cwirko M, Mierzecki A, Chlabicz S, Torres A, Almirall J, et al. Amoxicillin for acute lowerrespiratory-tract infection in primary care when pneumonia is not suspected: a 12-country, randomised, placebo-controlled trial. Lancet Infect Dis. 2013;13(2):123-9.

33. Huang DT, Yealy DM, Filbin MR, Brown AM, Chang CH, Doi Y, Donnino MW Fine J, Fine MJ, Fischer MA, et al. Procalcitonin-guided use of antibiotics for lower respiratory tract infection. N Engl J Med. 2018;379(3):236-49.

34. Mengel-Jorgensen T, Jensen MB. Variation in the use of point-of-care ultrasound in general practice in various European countries. Results of a survey among experts. Eur J Gen Pract. 2016;22(4):274-7.

35. Statistique des cabinets médicaux et des centres ambulatoires (MAS). Les cabinets médicaux et centres ambulatoires en 2015. https://www.bfs.admin. ch/bfs/fr/home/actualites/quoi-de-neuf.assetdetail.4924761.html. Accessed 23 Apr 2019

\section{Publisher's Note}

Springer Nature remains neutral with regard to jurisdictional claims in published maps and institutional affiliations.

Ready to submit your research? Choose BMC and benefit from:

- fast, convenient online submission

- thorough peer review by experienced researchers in your field

- rapid publication on acceptance

- support for research data, including large and complex data types

- gold Open Access which fosters wider collaboration and increased citations

- maximum visibility for your research: over $100 \mathrm{M}$ website views per year

At $\mathrm{BMC}$, research is always in progress.

Learn more biomedcentral.com/submissions 